Pacific Journal of Mathematics

$p$-ADIC INTEGRAL TRANSFORMS ON COMPAC 


\title{
p-ADIC INTEGRAL TRANSFORMS ON COMPACT SUBGROUPS OF $\mathbf{C}_{p}$
}

\author{
NeAl KoblitZ
}

Let $p$ be a fixed prime, and let $C_{p}$ denote the $p$-adic completion of the algebraic closure of $\mathbf{Q}_{p}$. For $d$ a fixed positive integer prime to $p$, set $X=X_{d}=\lim _{\leftarrow N-} \mathbf{Z} / d p^{N} \mathbf{Z}$. For example, $X_{1}=\mathbf{Z}_{p}$. We shall first discuss the "inverse Mellin" integral transform $f_{\mu}(\rho)=\int_{X} \rho(x) d \mu(x)$ for $\rho$ a $\mathbf{C}_{p}$-valued bounded measure on $X$. We then discuss a second type of $p$-adic integral transform, which to a continuous function $f(x)$ on $X$ associates the analytic function whose Taylor expansion coefficients are $f(n)$. Thirdly, for $\sigma$ a compact subset of $\mathbf{C}_{p}$ the $p$-adic Stieltjes transform $\varphi(z)=\int_{\sigma}(z-x)^{-1} d \mu(x)$ was shown by Barsky and Vishik to give a correspondence between measures $\mu$ on $\sigma$ and a certain class of analytic functions $\varphi$ on the complement of $\sigma$. We shall show that when $\sigma$ is a compact subgroup of $C_{p}$, the Stieltjes transform is closely related to the first two transforms. Some examples and arithmetic applications will also be discussed.

1. Let $p, \mathbf{C}_{p}$ and $X=X_{d}$ be as above. The $p$-adic absolute value in $\mathbf{C}_{p}$ is normalized so that $|p|_{p}=1 / p$. For $u \in \mathbf{C}_{p}$ with $|u|_{p}=1$, let $\bar{u}$ denote its residue in $F_{p}^{\text {algcl }}$, and let $\omega(u)$ be the Teichmüller representative of $u$, i.e., the unique root of unity of order prime to $p$ with the same residue in $F_{p}^{\text {algcl }}$. Set $\langle u\rangle=u / \omega(u)$. The ring $X$ is isomorphic to the product of rings $\mathbf{Z} / d \mathbf{Z}$ and $\mathbf{Z}_{p}$ under the two projections $\pi_{1}$ and $\pi_{2}$, where for $x \in X$ we set $\pi_{1}(x)=$ the image of $x$ modulo $d$ and $\pi_{2}(x)=$ the limit of the image of $x$ modulo $p^{N}$ ("forget $\bmod d$ information"). Let $a+d p^{N} \mathbf{Z}_{p}$ denote the set of $x \in X$ for which $x \equiv a \bmod d p^{N}$. Let $X^{m}=X_{d} \times \mathbf{Z}_{p}^{m-1}$ denote the product of $X$ with $m-1$ copies of $\mathbf{Z}_{p}$.

A function $f(n)$ mapping the nonnegative integers to $\mathbf{C}_{p}$ extends to a continuous function on $X$ if and only if for every $\varepsilon>0$ we have $\left|f\left(n_{1}\right)-f\left(n_{2}\right)\right|_{p}<\varepsilon$ whenever $n_{1} \equiv n_{2} \bmod d p^{N}$ for $N$ sufficiently large. In particular, for $u \in \mathbf{C}_{p}$ the function $f(n)=u^{n}$ extends to $X$ if and only if $\left|u^{d}-1\right|_{p}<1$. In that case $u^{x}=\omega(u)^{\pi_{1}(x)}\langle u\rangle^{\pi_{2}(x)}$.

Let $U_{1} \subset \mathbf{C}_{p}$ denote the open unit disc about 1 , and let $U_{d}=\{u \in$ $\left.C_{p}|| u^{d}-\left.1\right|_{p}<1\right\}$ denote the union of the open unit discs around the $d$ th roots of unity. Let $U^{m}=U_{d} \times U_{1}^{m-1}$. We say that a set $\left\{u_{1}, u_{2}, \ldots, u_{m}\right\}$ $\in U^{m}$ is (multiplicatively) $X^{m}$-independent if the relation $u_{1}^{x_{1}} u_{2}^{x_{2}} \cdots u_{m}^{x_{m}}=$ 1 for $x=\left(x_{1}, \ldots, x_{m}\right) \in X^{m}$ implies $x=0$. By replacing $u_{j}$ by $u_{j}^{d p^{N}}$ for 
some large $N$, one sees that a set is multiplicatively $X^{m}$-independent if and only if its $p$-adic logarithms are $\mathbf{Q}_{p}$-linearly independent.

Let $\boldsymbol{\sigma}$ be a compact subset of $\mathbf{C}_{p}^{*}=\mathbf{C}_{p}-\{0\}$. Suppose that $\sigma$ is a subgroup of $\mathbf{C}_{p}^{*}$. Then clearly $\sigma \subset U_{d}$ for some $d$. Choose $d$ to be minimal with $\sigma \subset U_{d}$. It is not hard to see that there exists a finite $X^{m}$-independent set $u=\left\{u_{1}, u_{2}, \ldots, u_{m}\right\}$ such that $\sigma=\sigma_{\text {tors, } p} u^{X^{m}}$, where

$$
u^{X^{m}}={ }_{\text {def }}\left\{u_{1}^{x_{1}} \cdots u_{m}^{x_{m}} \mid x_{1} \in X, x_{j} \in \mathbf{Z}_{p}(j>1)\right\}
$$

and $\sigma_{\text {tors, } p} \subset \sigma$ is the (finite) subgroup of $p$ th power roots of unity. For some finite $N_{0}$ any $u \in \sigma$ can be written uniquely in the form $u=\zeta u_{1}^{x_{1}}$ $\cdots u_{m}^{x_{m}}$ with $x \in X^{m}$ and $\zeta^{p_{0}}=1$. We say that $\sigma$ has no $p$-torsion if $\sigma_{\text {tors, } p}=\{1\}$.

Let $\rho$ denote a (continuous) one-dimensional representation of $X^{m}$ in $\mathrm{C}_{p}$. The image $\rho\left(X^{m}\right) \subset \mathbf{C}_{p}^{*}$ is a compact subgroup; it has no $p$-torsion if $\rho$ is faithful.

Let $\delta_{j} \in X^{m}$ be the $m$-tuple with 1 in the $j$ th place and 0 everywhere else. Then the map $\rho \mapsto\left(\rho\left(\delta_{1}\right), \ldots, \rho\left(\delta_{m}\right)\right)$ gives a one-to-one correspondence between one-dimensional representations of $X^{m}$ and $U^{m}$. For $u=\left(u_{1}, \ldots, u_{m}\right) \in U^{m}$, we sometimes let $\rho_{u}$ denote the representation such that $\rho_{u}\left(\delta_{j}\right)=u_{j}$. Note that $\rho_{u}$ is faithful if and only if $u$ is $X^{m}$-independent.

Let $\mu$ be a measure on $X^{m}$, i.e., a bounded finitely additive map $U \mapsto \mu(U)$ from compact-open subsets $U \subset x^{m}$ to $\mathbf{C}_{p}$.

Definition. If $\mu$ denotes a measure on $X^{m}$ and $\rho$ denotes a representation of $X^{m}$ in a finite dimensional $\mathbf{C}_{p}$-vector space, then the map

$$
(\mu, \rho) \mapsto f_{\mu}(\rho)=\int_{X^{m}} \rho(x) d \mu(x)
$$

is called the $p$-adic inverse Mellin transform of $\mu$.

REMARKs. 1 . The terminology comes by analogy with the transform $g_{f}(x)=\int x^{s} f(s) d s$ which is inverse to the Mellin transform $f(s)=$ $\int x^{s} g(x) d x / x$. Here the characters of $\mathbf{R}$ are parametrized by $x$. In addition, this definition generalizes the construction used by Hà Huy Khoái [5] to invert the $p$-adic Mellin-Mazur transform.

2. If $m=1$ and $\rho$ is a faithful one-dimensional representation of $X_{d}$, then this integral can be viewed as a Mellin-Mazur transform by a change of variables. Namely, we fix the image $\sigma$ of $\rho_{1}$, and we let $\rho$ vary over representations with image contained in $\sigma$. If we set $u_{1}=\rho_{1}(1)$, so that 
$\sigma=u_{1}^{X_{d}}$, then such $\rho$ are parametrized by $y \in X_{d}$, that is, $\rho_{y}=\rho_{1}^{y}$ : $x \mapsto u_{1}^{x y}$. Finally, let $\nu$ be the measure on $\sigma$ obtained by pulling back $\mu$ : $d \nu\left(u_{1}^{x}\right)=d \mu(x)$. In this situation

$$
f_{\mu}\left(\rho_{1}^{y}\right)=\int_{X^{m}} u_{1}^{x y} d \mu(x)=\int_{\sigma} x^{y} d \nu(x)=L_{\nu}(y),
$$

which is the $p$-adic $L$-function corresponding to the measure $\nu$ on $\sigma$.

THEOREM 1. The inverse Mellin transform $f_{\mu}\left(\rho_{u}\right)$ of a measure $\mu$ on $X^{m}$ is a bounded analytic function of $u \in U^{m}$, and any bounded analytic function on $U^{m}$ is the inverse Mellin transform of some measure.

Proof. Clearly the map

$$
u=\left(u_{1}, \ldots, u_{m}\right) \mapsto f_{\mu}\left(\rho_{u}\right)=\int_{X^{m}} u_{1}^{x_{1}} \cdots u_{m}^{x_{m}} d \mu\left(x_{1}, \ldots, x_{m}\right)
$$

is bounded and analytic. To go the other way, given $f$ we define

$$
\mu_{f}\left(a+d p^{N} X^{m}\right)=\frac{1}{d p^{N}} \sum_{\xi} \xi^{-a} f(\xi),
$$

where $a+d p^{N} X^{m}$ denotes the compact-open subset

$$
a_{1}+d p^{N_{1}} \mathbf{Z}_{p} \times a_{2}+p^{N_{2}} \mathbf{Z}_{p} \times \cdots \times a_{m}+p^{N_{m}} \mathbf{Z}_{p} \subset X^{m}
$$

in the notation $p^{N}$ on the right $N$ denotes $N_{1}+\cdots+N_{m}$; the sum on the right is over all $\xi=\left(\xi_{1}, \ldots, \xi_{m}\right) \in U^{m}$ for which $\xi_{1}^{d p^{N_{1}}}=\xi_{2}^{p^{N_{2}}}=\cdots=$ $\xi_{m}^{p^{N_{m}}}=1$; and $\xi^{-a}$ denotes $\Pi \xi_{j}^{-a_{j}}$. Clearly the mapping $\mu_{f}$ defined by (1.3) on the usual basis of compact-open subsets of $X^{m}$ extends to an additive function of compact-open subsets; it is not hard to show that $\mu_{f}$ is bounded, using the analyticity and boundedness of $f$. We claim that $f(u)=\int u^{x} d \mu(x)$ for any $u \in U^{m}$. Since $f(u)$ can be approximated by a finite linear combination of monomials in $\left(\left\langle u_{1}\right\rangle, u_{2}, \ldots, u_{m}\right) \in U_{1}^{m}$ multiplied by the characteristic function with respect to $u_{1}$ of one of the $d$ unit discs in $U_{d}$, it suffices to check the claim in the case when $f(u)$ is such a function. But in this case the desired equality is proved in a standard way, essentially by orthogonality of characters on $\mathbf{Z} / d p^{N_{1}} \mathbf{Z} \times \mathbf{Z} / p^{N_{2}} \mathbf{Z} \times \cdots$ $\times \mathbf{Z} / p^{N m} \mathbf{Z}$.

REMARKS. 1. In the case $m=1$, Hà Huy Khoái proves a more general theorem, namely that the so-called $h$-admissible distributions $\mu$ correspond to all functions on $U_{d}$ which grow more slowly than $\left(\log _{p} u\right)^{h}$ as $u$ approaches the boundary of $U_{d}$. In particular, for $h=1$ the same construction (1.3) of the measure applies. The point is that, like a bounded 
analytic function, an analytic function which grows more slowly than $\log _{p}$ is determined by its values at the roots of unity $\xi$.

2. A conjecture of $\mathrm{R}$. Greenberg asserts that for any $X^{m}$-independent set $u \in U^{m}$, a bounded analytic function on $U^{m}$ (with coefficients in $\mathbf{Z}_{p}$ ) is determined by its values on $u^{y}$ as $y$ varies over $X_{d}$, where $u^{y}$ denotes $\left(u_{1}^{y}, u_{2}^{\pi_{2}(y)}, \ldots, u_{m}^{\pi_{2}(y)}\right)$. Equivalently, the conjecture is that, if $\rho$ is a faithful one-dimensional representation of $X^{m}$ and if $\int_{X^{m}} \rho(x y) d \mu(x)=0$ for $y \in X_{d}$, then $\mu \equiv 0$.

2. We now let $m=1$, and consider higher dimensional continuous representations of $X=X_{d}=\lim _{\leftarrow N-} \mathbf{Z} / d p^{N} \mathbf{Z}$. If $\rho_{1}$ is an irreducible representation of $X$ in an $n$-dimensional $C_{p}$-vector space, then $\rho_{1}(1)$ has a single eigenvalue $v_{1}$, and $\rho_{1}(x)$ has eigenvalue $v_{1}^{x}$. Note that $v_{1} \in U_{d}$. For $\mu$ a measure on $X$, let $f_{\mu}\left(\rho_{1}\right)$ be defined by (1.1), and let $\nu$ be the measure on $\sigma=v_{1}^{X}$ defined by $d \nu\left(v_{1}^{x}\right)=d \mu(x)$. Now define $L_{\nu}(y)$ by the MellinMazur transform: $L_{\nu}(y)=\int_{\sigma} x^{y} d \nu(x)$.

THEOREM 2. With these assumptions and notation, when $f_{\mu}\left(\rho_{1}\right) \neq 0$ the order of zero of $L_{\nu}(y)$ at $y=1$ is equal to the co-rank of $f_{\mu}\left(\rho_{1}\right)$.

Proof. Let $V_{1}=\rho_{1}(1)$, and let $V=C V_{1} C^{-1}$ be the Jordan normal form. Since $\rho_{1}$ is irreducible, it follows that $V$ is a single $n \times n$ Jordan block. Thus, $V=v_{1}+\varepsilon$, where $v_{1}=v_{1} J$ is a scalar matrix and $\varepsilon$ denotes the matrix with ones just above the main diagonal and zeros elsewhere. Then

$$
f_{\mu}\left(\rho_{1}\right)=\int_{X} V_{1}^{x} d \mu(x)=C^{-1} \int_{X}\left(v_{1}+\varepsilon\right)^{x} d \mu(x) C .
$$

Thus, the co-rank of $f_{\mu}\left(\rho_{1}\right)$ is the same as that of

$$
\begin{aligned}
\sum_{j=0}^{n-1} \varepsilon^{j} \int_{X}\left(\begin{array}{l}
x \\
j
\end{array}\right) v_{1}^{x-j} d \mu(x) & =\left.\sum_{j=0}^{n-1} \frac{1}{j !} \varepsilon^{j}\left(\frac{d}{d v}\right)^{j} \int_{X} v^{x} d \mu(x)\right|_{v=v_{1}} \\
& =\sum_{j=0}^{n-1} \frac{g^{(j)}\left(v_{1}\right)}{j !} \varepsilon^{j},
\end{aligned}
$$

where $g(v)=\int_{X} v^{x} d \mu(x)$. Making the change of variables $v=v_{1}^{y}$, we have

$$
g\left(v_{1}^{y}\right)=\int_{X} v_{1}^{y x} d \mu(x)=\int_{\sigma} x^{y} d \nu(x)=L_{\nu}(y) .
$$

Let $r$ be the order of zero of $L_{\nu}(y)$ at $y=1$. Then $L_{\nu}(1)=L_{\nu}^{\prime}(1)=\cdots=$ $L_{\nu}^{(r-1)}(1)=0, L_{\nu}^{(r)}(1) \neq 0$, and so $g\left(v_{1}\right)=g^{\prime}\left(v_{1}\right)=\cdots=g^{(r-1)}\left(v_{1}\right)=0$, 
$g^{(r)}\left(v_{1}\right) \neq 0$. Then $f_{\mu}\left(\rho_{1}\right)$ has the same co-rank as $\sum_{j=r}^{n-1} g^{(j)}\left(v_{1}\right) / j ! \varepsilon^{j}$, where $r<n$, because $f_{\mu}\left(\rho_{1}\right) \neq 0$. But the latter co-rank is obviously $r$.

3. Let $\bar{U}_{d}=\left\{u \in \mathbf{C}_{p}|| u^{d}-\left.1\right|_{p} \geq 1\right\}$ denote the complement of $U_{d}$, and set $\bar{U}^{m}=\bar{U}_{d} \times \bar{U}_{1}^{m-1}$. For any $z=\left(z_{1}, \ldots, z_{m}\right) \in \bar{U}^{m}$, let $\mu_{z}$ denote the bounded measure on $X^{m}$ which is defined on the standard basis of compact-open sets by

$$
\mu_{z}\left(a+d p^{N} X^{m}\right)=\frac{z^{a}}{\left(1-z_{1}^{d p^{N_{1}}}\right)\left(1-z_{2}^{p^{N_{2}}}\right) \cdots\left(1-z_{m}^{p^{N_{m}}}\right)},
$$

where the notation $a+d p^{N} X^{m}$ has the same meaning as in (1.3), except that we agree to take the representatives $a_{j}$ in the range $0 \leq a_{1}<d p^{N_{1}}$, $0 \leq a_{j}<p^{N_{j}}(j>1)$, and $z^{a}$ denotes $\Pi z_{j}^{a}$. (It is easy to check that this $\mu_{z}$ actually extends to a bounded measure on $X^{m}$.)

THEOREM 3. For any continuous function $f: X^{m} \rightarrow \mathbf{C}_{p}$, the transform

$$
g(z)=\int_{X^{m}} f(x) d \mu_{z}(x), \quad z \in \bar{U}^{m},
$$

has the properties

(1) $g(z)$ is bounded and Krasner analytic in each $z$, on $\bar{U}^{m}$;

(2) $g(z) \rightarrow 0$ as $\left|z_{j}\right|_{p} \rightarrow \infty$ for each variable $z_{J}$ with any fixed values of the remaining variables;

(3) in the open unit polydisc $\left|z_{j}\right|_{p}<1, g(z)$ has the expansion $\sum f(n) z^{n}$, where $n=\left(n_{1}, \ldots, n_{m}\right)$ runs through all $m$-tuples of nonnegative integers;

(4) for $\left|z_{j}\right|_{p}>1, j=1, \ldots, m, g(z)$ has the expansion $-\sum f(-n) z^{-n}$, where $n$ runs through all $m$-tuples of positive integers.

Conversely, if $g$ is any function satisfying (1) and (2), and if $g(z)=$ $\sum a_{n} z^{n}$ is its expansion in the open unit polydisc, then the sequence $f(n)=a_{n}$ extends to a continuous function on $X^{m}$, and we have (3.1) and also property (4).

Proof. This is essentially a theorem of Amice and Vélu [1] when $m=1$ (see the Appendix to [8] for a treatment using the measure $\mu_{z}$ ), and the general case is handled in the same way.

EXAMPLES. 1. For fixed $u \in U^{m}$, the transform of the representation $\rho_{u}$ (in the notation of $\S 1$ ) is simply $g(z)=\int_{X^{m}} u^{x} d \mu_{z}(x)=\prod_{j}\left(1-u_{j} z_{j}\right)^{-1}$.

2. Let $m=1$. According to results of Katz [4], a $p$-adic modular form $F$ of weight zero (and level 1) can be written as a function of the $j$-invariant which is Krasner analytic outside of small discs around the 
supersingular points. Let $\left\{\bar{s}_{i}\right\} \subset F_{p}^{\text {algcl }}$ be the residues of all supersingular values of $j$. It is known that in fact $\left\{\bar{s}_{i}\right\} \subset F_{p^{2}}$ (for a table of $\bar{s}_{i}$ for $p \leq 307$, see [10]). Suppose that $j=0$ is not supersingular, i.e., $p \equiv 1 \bmod 6$. Let $F_{\infty}$ be the value at the cusp. Then $F-F_{\infty}=g(j)$ satisfies properties (1) and (2) of Theorem 3, with $j$ playing the role of the variable $z$. Here $d$ is some divisor of $p^{2}-1$, since $\bar{s}_{i}^{p^{2}-1}=1$ for each $i$. Thus, if $F(j)=F_{\infty}$ $+\sum_{n=0}^{\infty} a_{n} j^{n}$ for $|j|_{p}<1$, the coefficients $f(n)=a_{n}$ extend to a continuous function on $X_{d}$, and

$$
F(j)=F_{\infty}+\int_{X_{d}} f(x) d \mu_{j}(x), \quad j \in \bar{U}_{d} .
$$

In addition,

$$
F(j)=F_{\infty}-\sum_{n=1}^{\infty} f(-n) j^{-n} \text { for }|j|_{p}>1 .
$$

Hence, we have congruences for the $j$ - and $1 / j$-expansion coefficients which generalize those in Ashworth [2] and Koblitz [6].

4. We now discuss a third type of integral transform. Let $\rho: X^{m} \rightarrow U_{d}$ be a one-dimensional continuous representation, as in $\$ 1$, and let $\rho_{j}$ denote the $j$ th component, i.e., $\rho_{j}\left(x_{1}, \ldots, x_{m}\right)=\rho\left(0, \ldots, 0, x_{j}, 0, \ldots, 0\right)$. Let $\mu$ be a bounded measure on $X^{m}$. For $z \in \mathbf{C}_{p}^{m}$ with $z_{j}$ in the complement of the image of $\rho_{j}$, in particular for $z \in \vec{U}^{m}$, we define the Stieltjes transform of $\rho$ and $\mu$ as follows:

$$
\psi_{\rho, \mu}(z)=\int_{X^{m}} \frac{d \mu(x)}{\prod_{j=1}^{m}\left(1-z_{j} \rho_{j}(x)\right)} .
$$

The next theorem gives a relation between the three transforms in $\S \S 1,3$ and 4.

THEOREM 4. Let $\mu$ be a measure on $X^{m}$, and let $\rho$ be a one-dimensional representation of $X^{m}$ in $\mathbf{C}_{p}^{*}$. Let $f_{\mu}(\rho)$ be the inverse Mellin transform defined by (1.1). For $y \in X_{d}$, let $\rho^{y}$ denote the representation $\rho^{y}(x)=\rho(x y)=$ $\rho\left(x_{1} y, x_{2} \pi_{2}(y), \ldots, x_{m} \pi_{2}(y)\right)$. If the transform (3.1) associated to the measure $\mu_{z}$ for $z \in \bar{U}^{m}$ is applied to the function $y \mapsto f_{\mu}\left(\rho^{y}\right)$, then the result is the Stieltjes transform $\psi_{\rho, \mu}(z)$.

Proof.

$$
\begin{aligned}
\int_{X^{m}} f_{\mu}\left(\rho^{y}\right) d \mu_{z}(y) & =\int_{X^{m}} \int_{X^{m}} \rho^{y}(x) d \mu(x) d \mu_{z}(y) \\
& =\int_{X^{m}} \int_{X^{m}} \rho^{y}(x) d \mu_{z}(y) d \mu(x) .
\end{aligned}
$$


But

$$
\int_{X^{m}} \rho(x y) d \mu_{z}(y)=\prod_{j} \int \rho_{j}(x)^{y} d \mu_{z_{j}}(y)=\prod_{j}\left(1-z_{j} \rho_{j}(z)\right)^{-1},
$$

and so

$$
\int_{X^{m}} f_{\mu}\left(\rho^{y}\right) d \mu_{z}(y)=\int_{X^{m}} \frac{d \mu(x)}{\prod_{j}\left(1-z_{j} \rho_{j}(x)\right)},
$$

as claimed.

REMARKS. 1. When $m=1$, our $\psi$ in (4.1) is essentially the transform $\varphi_{\nu}(z)=\int_{\sigma}(z-x)^{-1} d \nu(x), z \in \bar{\sigma}$, that is studied in [3], [12] (see also the Appendix to [8]). Namely, $\psi_{\rho_{u}, \mu}(z)=z^{-1} \varphi_{\nu}\left(z^{-1}\right)$, where $\nu\left(u^{x}\right)=d \mu(x)$. Barsky and Vishik have shown that any Krasner analytic function on $\bar{\sigma}$ which vanishes at infinity and which grows more slowly than $1 / \operatorname{dist}(z, \sigma)$ as $z \rightarrow \sigma$ is of the form $\varphi(z)$. On the other hand, if $\sigma \subset U_{d}$ and $z \in \bar{U}_{d}$, then such a function of $z$ can also be written in the form $\int_{X_{d}} f(x) d \mu_{z}(x)$, with $f$ the continuous function which interpolates the Taylor expansion coefficients. Theorem 4 says that, because our function of $z$ is actually analytic on $\bar{\sigma}$ (not only on $\bar{U}_{d}$ ) and $\sigma$ is a compact subgroup of $\mathbf{C}_{p}^{*}$, it follows that $f$ extends to an analytic function on $U_{d} \supset \sigma=u^{X_{d}}$ (not just a continuous function on $\sigma$ ) and so is given by the inverse Mellin transform of a measure.

2. Theorem 4 is the $p$-adic analog of the fact that the classical Stieltjes transform is the square of the Laplace transform $L(f)=\int_{0}^{\infty} e^{-x y} f(x) d x$. Compare the proof of Theorem 4 with the relation (in which we think of $e^{-z y} d y$ as $\left.d \mu_{z}(y)\right)$ :

$$
L(L(f))(z)=\int_{0}^{\infty} \int_{0}^{\infty} e^{-x y} f(x) d x\left(e^{-z y} d y\right)=\int_{0}^{\infty}(z+x)^{-1} f(x) d x .
$$

\section{REFERENCES}

[1] Y. Amice and J. Vélu, Distributions p-adiques associées aux séries de Hecke, Journées Arith., 1974.

[2] M. H. Ashworth, Congruence properties of coefficients of modular forms using sigma functions, Ph.D. thesis (Oxford University, 1966).

[3] D. Barsky, Transformation de Cauchy p-adique et algébre d'Iwasawa, Math. Ann., 232 (1978), 255-266.

[4] N. M. Katz, p-adic Properties of Modular Schemes and Modular Forms, Proc. 1972 Antwerp Summer School, Springer Lectures Notes in Math., 350 (1973), 70-189.

[5] Hà Huy Khoái, Inverse formula for the Mellin-Mazur transform and some applications, to appear. 
[6] N. Koblitz, 2-adic and 3-adic ordinals of $(1 / j)$-expansion coefficients for the weight 2 Eisenstein series, Bull. London Math. Soc., 9 (1977), 188-192.

[7] _ - p-adic Numbers, p-adic Analysis, and Zeta-Functions, Springer-Verlag, 1977.

[8] p-adic Analysis: a Short Course on Recent Work, Cambridge Univ. Press, 1980.

[9] B. Mazur, Analyse p-adique, Bourbaki report (unpublished), 1972.

[10] B. Mazur and H. P. F. Swinnerton-Dyer, Arithmetic of Weil curves, Inventiones Math., 25 (1974), 1-61.

[11] Modular Functions in One Variable IV, Springer Lecture Notes in Math., 476, Springer-Verlag, 1975.

[12] M. M. Vishik, On applications of the Shnirelman integral in non-archimedean analysis, Uspekhi Mat. Nauk, 34 (1979), 223-224.

Received February 23, 1984.

UNIVERSITY OF WASHINGTON

SEATTLE, WA 98195 


\section{PACIFIC JOURNAL OF MATHEMATICS EDITORS}

V. S. VARAdarajan (Managing Editor)
University of California
Los Angeles, CA 90024
CHARLES R. DePrima
California Institute of Technology
Pasadena, CA 91125
R. FINN
Stanford University
Stanford, CA 94305

V. S. VARADARAJAN (Managing Editor)

Hermann FlaschKa

University of Arizona

Tucson, AZ 85721

RAMESH A. GANGOlli

University of Washington

Seattle, WA 98195

ROBION KIRBY

University of California

Berkeley, CA 94720
C. C. MOORE

University of California

Berkeley, CA 94720

H. SAMELSON

Stanford University

Stanford, CA 94305

Harold Stark

University of California, San Diego

La Jolla, CA 92093

\section{ASSOCIATE EDITORS}
R. ARENS
E. F. BECKENBACH
B. H. NeumanN
F. WOLF
K. Yoshida (1906-1982)

\section{SUPPORTING INSTITUTIONS}

\author{
UNIVERSITY OF ARIZONA \\ UNIVERSITY OF BRITISH COLUMBIA \\ CALIFORNIA INSTITUTE OF TECHNOLOGY \\ UNIVERSITY OF CALIFORNIA \\ MONTANA STATE UNIVERSITY \\ UNIVERSITY OF NEVADA, RENO \\ NEW MEXICO STATE UNIVERSITY \\ OREGON STATE UNIVERSITY
}

UNIVERSITY OF OREGON UNIVERSITY OF SOUTHERN CALIFORNIA

STANFORD UNIVERSITY

UNIVERSITY OF HAWAII

UNIVERSITY OF TOKYO

UNIVERSITY OF UTAH

WASHINGTON STATE UNIVERSITY

UNIVERSITY OF WASHINGTON 


\section{Pacific Journal of Mathematics}

\section{Vol. 120, No. $1 \quad$ September, 1985}

Ulrich F. Albrecht, A note on locally $A$-projective groups $\ldots \ldots \ldots \ldots \ldots 1$

Marilyn Breen, A Krasnosel'skiü-type theorem for unions of two starshaped

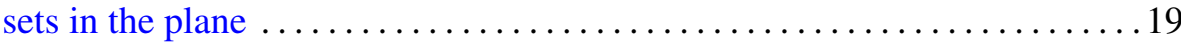

Anthony Carbery, Sun-Yung Alice Chang and John Brady Garnett,

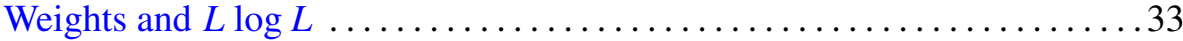

Joanne Marie Dombrowski, Tridiagonal matrix representations of cyclic

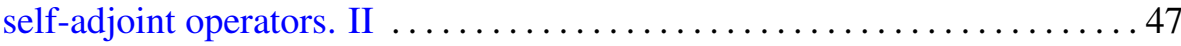

Heinz W. Engl and Werner Römisch, Approximate solutions of nonlinear random operator equations: convergence in distribution $\ldots \ldots \ldots \ldots \ldots 55$

P. Ghez, R. Lima and J. E. Roberts, $W^{*}$-categories $\ldots \ldots \ldots \ldots \ldots \ldots$

Barry E. Johnson, Continuity of homomorphisms of Banach $G$-modules . . 111 Elyahu Katz and Sidney Allen Morris, Free products of topological groups

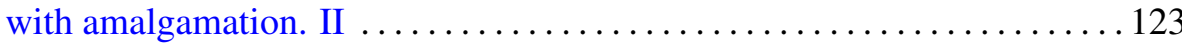

Neal I. Koblitz, $p$-adic integral transforms on compact subgroups of $\mathbf{C}_{p} \ldots 131$ Albert Edward Livingston, A coefficient inequality for functions of positive real part with an application to multivalent functions $\ldots \ldots \ldots 139$

Scott Carroll Metcalf, Finding a boundary for a Hilbert cube manifold

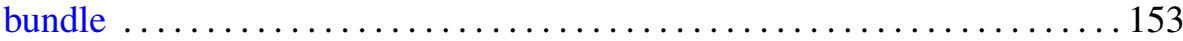

Jack Ray Porter and R. Grant Woods, When all semiregular $H$-closed extensions are compact ........................

Francisco José Ruiz and José Luis Torrea, A unified approach to Carleson measures and $A_{p}$ weights. II $\ldots \ldots \ldots \ldots \ldots \ldots \ldots \ldots \ldots \ldots \ldots \ldots \ldots$

Timothy DuWayne Sauer, The number of equations defining points in general position

John Brendan Sullivan, Universal observability and codimension one subgroups of Borel subgroups

Akihito Uchiyama, Extension of the Hardy-Littlewood-Fefferman-Stein inequality 